\title{
Smart Risk Assessment Systems using Belief-rule-based DSS and WSN Technologies
}

\author{
Karl Andersson \\ Pervasive and Mobile Computing Laboratory \\ Luleå University of Technology \\ SE-931 87 Skellefteå, Sweden \\ karl.andersson@1tu.se
}

\author{
Mohammad Shahadat Hossain \\ Department of Computer Science and Engineering \\ University of Chittagong \\ University-4331, Bangladesh \\ hossain_ms@cu.ac.bd
}

\begin{abstract}
Smart risk assessment systems are becoming more and more important in the society. If the chances of reducing and managing certain [[risks are increased, the impacts can be controlled and reduced significantly. This article surveys different belief-rule-based decision support systems and various wireless sensor network technologies that can be used in collaboration to build interesting risk assessment applications. We propose a model for building such an environment and describe a potential application of our proposed model for assessing flood risks in a case study.
\end{abstract}

Keywords-Smart risk assessment systems; Belief-rule-based DSS; WSN; Flood risk assessment

\section{INTRODUCTION}

Risk assessment of flooding is important because flooding is considered to be one of the most catastrophic forms of natural disaster, responsible for the highest number of fatalities and greater economic damage in comparison to other natural disasters [1][2]. Flooding involves complex, interrelated and multi-dimensional geophysical processes and has a wide range of impact on human lives, activities and properties. Furthermore, the risks of flooding on a specific object may force a series of further objects to suffer the consequences of flood disasters in a ripple fashion. In general, assessment of flood risk, like other risks, comprises different stages including identification, modeling and evaluation [2][3][4]. The identification stage is concerned with characterizing factors that cause a flood and the physical and the socio-economic objects exposed to flooding. The modeling stage is associated with the estimation of likelihood of a flood and its consequences to obtain a calculated view of risk. The evaluation stage determines the acceptance or aversion limit of flood risk, based on which decisions should be taken to reduce the consequences of flooding. Each decision should be analyzed against environmental, social and economic criteria, to make it a balanced one. Wireless sensor networks (WSNs) are used to collect and distribute sensor data, often processed by data mining techniques, which can be used to collect various data necessary to assess the risks of flooding.

This article surveys different belief-rule-based decision support systems and various wireless sensor network technologies that can be used in collaboration to build interesting risk assessment applications. We propose a model for building such an environment and describe a potential application of our proposed model for assessing flood risks in a case study.

The remainder of this article is structured the following way: Section II surveys the area of smart risk assessment systems, while Section III describes impacts from belief-rulebased decision support system (DSS). Section IV describes impacts from WSN technologies, while Section V presents a case study where our model is applied to flood risk assessment. Finally, Section VI concludes the article and indicates future work.

\section{SMART RISK ASSESSMENT SYSTEMS}

Existing methods of flood risk assessment are focused on the analysis of exposure units to flooding, probability of flood occurrence, flood damage assessment or a combination of both $[19][20][21]$. Some methods calculate the likelihood of occurrence of flooding by taking the flood depth dimension into account [8][22]. In other methods, depth and areal dimensions are considered along with flood causing and intensifying factors such as rainfall, topography and river characteristics. All the above-mentioned methods have relied upon quantitative measures. However, in most situations where places are identified as at risk of flooding, there is little data available with which to estimate either the probability of exceedence of a given discharge or to predict the depths and velocities resulting from such a discharge. Flood frequency estimates, whether estimated by fitting statistical distributions, by regionalization from gauged sites, or by continuous simulation [23][24] are known to be highly uncertain. The predictions of flood inundation models, even when conditioned on observed depth or flooded area information, are also known to be uncertain (and in some cases cannot match the observations throughout the flow domain) [25]. In the past, these quantitative uncertainties have often been ignored in flood risk assessments but should be taken into account in the future. This recognition has been a major driver for the UK FRMRC program and the science program of the international Prediction of Ungauged Basins (PUB) initiative. In addition, some of the factors contributing to flood likelihood are qualitative in nature and difficult to quantify, in part because of 
the ill-defined nature of the problem. Hence, expert judgments are needed for identification of the values of some variables [5][9]. Many of the 'softer' social dimensions, which influence flood risk, are also difficult to quantify, for example the effects of public behavior and policy. Whilst it has been common practice to use contingent valuation to quantify economic impacts, such techniques are controversial when applied as 'willingness to pay' measures to attempt to quantify other social impacts such as disruption, stress and wider community impacts. Meanwhile, more inclusive social science concepts such as social vulnerability and adaptive capacity have been developed by social scientists, but these concepts have largely remained as qualitative concepts and rarely been used by the flood risk modelers [6][7][8]. Hence, there is a lack of a consistent and unified framework for bringing together the 'hard' physical and economic dimensions with the 'softer' social dimensions, and of aggregating both qualitative and quantitative data with uncertainty.

\section{IMPACTS OF BELIEF-RULE-BASED EXPERT SYSTEMS}

A Belief Rule Base (BRB) is a knowledge representation schema, which allows the capturing of various types of uncertain information. Evidential Reasoning (ER) is used as the inference methodology in the Belief Rule Based Expert System [11][12][13][14][15][16][17]. ER is mainly used to aggregate the rules in the BRB either in recursive or analytical way. This approach is widely known as RIMER methodology. A BRB can capture nonlinear causal relationships under uncertainty between antecedent attributes and the consequent, which is not possible in traditional IF-THEN rules.

Other knowledge representation languages such as Propositional Logic (PL), First-order Logic (FOL), and Fuzzy Logic (FL) are used to develop the knowledge-base [18][19] and reasoning mechanisms such as forward-chaining (FC) and backward chaining (BC) are used to develop inference engines [20]. However, neither PL nor FOL are equipped with schemas that can capture uncertainties. On the other hand, although FL handles some uncertainties due to ambiguity, vagueness or imprecision, it can't handle other types of uncertainties such as ignorance, incompleteness, and ignorance in fuzziness that may exist with different categories of variables associated with e-government. However, RIMER methodology as mentioned can handle different types of uncertainties that exist either in knowledge representation or in inference mechanism [21][22]. Fig.1 shows the architecture of the Belief Rule Based System, consisting of its input, inference procedures and output components. Inference procedures consist of input transformation, rule activation weight calculation, rule update mechanisms, followed by the aggregation of the rules of a BRB by using ER. This aggregation allows obtaining the distribution of belief degrees for the consequent (C) attribute for the given values of antecedent attributes (input data) of a BRB $\left(\boldsymbol{P}_{\boldsymbol{i}}\right)$ as illustrated in Fig. 1. The RIMER methodology has been employed to develop expert systems in the area of risk assessment, medical domain, meditation [21][22][23][24]. However, the capturing of input data on a real time basis will allow the estimation or prediction of an event either in risk assessment or medical domain more accurately. This will eventually enable the development of appropriate preparedness plan to tackle an event in advance. Therefore, the employment of the state of the art wireless sensors to collect the input data with an objective to feed the BRB system is necessary.

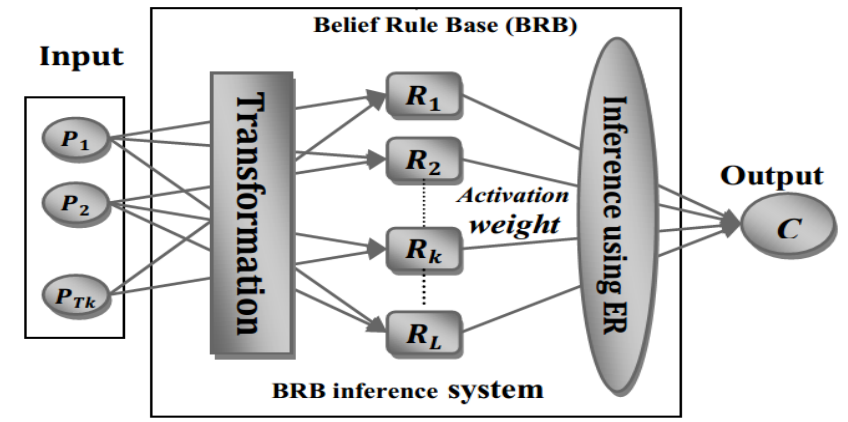

Fig. 1. Single-layer BRB inference architecture with RIMER Methodology

\section{IMPACTS OF WSN TECHNOLOGIES}

WSNs have been studied the past 15 years. Akyildiz et al. [26] provided a comprehensive overview and identified a few grand challenges including integration of sensor networks with the Internet as well as real-time and multimedia communication. A number of MAC protocols for wireless sensor networks have been proposed. Ye et al. [27] proposed SMAC while Jamieson et al. [28] proposed Sift. Demirkol et al. [29] surveyed the field of MAC protocols for wireless sensor networks. Also, a number of routing protocols for wireless sensor networks have been proposed. Manjeshwar et al. [30] proposed TEEN, while Schurgers et al. [31] proposed an energy efficient routing scheme. Al-Karaki et al. [32] and Akkaya et al. [33] surveyed the field of routing in wireless sensor networks. Perrig et al. [34] covered security in wireless sensor networks. The most common wireless technology used in wireless sensor networks is the IEEE 802.15.4 standard [35] while the 6LOWPAN architecture [36][37] was designed in order to allow for IPv6 packets to be carried on top of lowpower wireless networks, specifically IEEE 802.15.4. The most important standard for services is the Open Services Gateway initiative (OSGi) framework [38]. Kächele et al. [39] described a POSIX compliant implementation while Schmidt et al. [40] described a cloud-enabled implementation. The Session Initiation Protocol (SIP) [41] is an application layer protocol that was originally designed for establishing, maintaining, and closing multimedia sessions on the Internet. Rosenberg [42] and Lonnfors et al. [43] extended SIP for conveying presence information, also described by Schulzrinne in [44]. The use of SIP in wireless sensor networks was proposed by Roychowdhury et al. [45]. The Application Protocol for Constrained Networks/Nodes (CoAP) [46][47] is yet another application layer protocol that is intended for use in resourceconstrained Internet devices, such as nodes in wireless sensor networks. It was designed to easily translate to HTTP (Hypertext Transmission Protocol) for simplified integration with the web, while also meeting specialized requirements such as multicast support, very low overhead, and simplicity. CoAP can run on most devices that support UDP (User Datagram Protocol). 
Studies with WSNs being used to feed DSSs with input are described in [48][49]. However, these studies do not take into account belief-rule-based DSSs and the use of heterogeneous wireless sensor networking technologies to the potential being possible today. Furthermore, Hughes et al. [50][51] proposed grid-based flood prediction, while De Roure et al. [52] proposed flood warning times being improved by using pervasive and grid computing. Our proposed project uses cost efficient and open source-based software which is easier to maintain and easier to deploy. Furthermore, our proposed project takes more factors into account such as climatological (rainfall and rain fall duration), topographical (slope and aspect), geology (soil type, soil infiltration rate) river characteristics (river flow, river depth, river width, river bed slope) and human activities (land use, deforestation, embankment failure and many others). Also, our proposed project will develop procedures for transforming raw data received from sensors using advanced data mining techniques in a way that can be fed in the rule based expert DSS. This will address the issues of various types of uncertainties associated with the above-mentioned data.

\section{PROPOSED SYSTEM ARCHITECTURE TO ASSESS RisK OF FLOODING}

Highly complex systems are faced with increasing inability to cope with high impact events such as natural or man-made disasters and market disruptions. The assessment of risk for complex systems has become increasingly important. Risk is defined as a function of the probability of occurrence of an event and its consequences, which can be material, environmental or cultural damages. Risk assessment of flooding is important because flooding is considered to be one of the most catastrophic forms of natural disaster, responsible for the highest number of fatalities and greater economic damage in comparison to other natural disasters. Flooding involves complex, interrelated and multi-dimensional geophysical processes and has a wide range of impact on human lives, activities and properties. Furthermore, the risks of flooding on a specific object may force a series of further objects to suffer the consequences of flood disasters in a ripple fashion. In general, assessment of flood risk, like other risks, comprises different stages including identification, modeling and evaluation [1][2][3][4][5][6]. The identification stage is concerned with characterizing factors that cause a flood and the physical and the socio-economic objects exposed to flooding. The modeling stage is associated with the estimation of likelihood of a flood and its consequences to obtain a calculated view of risk. The evaluation stage determines the acceptance or aversion limit of flood risk, based on which decisions should be taken to reduce the consequences of flooding [7][8][9][10]. However, this paper presents the belief rule base framework to estimate the flood water level of an area as shown in Fig. 2. The framework considers meteorological, geological, river characteristics, topographical and human activities. 17 input data are required to feed the system, which are both quantitative and qualitative. Examples of quantitative data are rainfall, velocity, soil infiltration rate, etc. and the examples of qualitative data are settlement on the flood prone area as shown in Fig. 2. Various types of wireless sensors can be employed to collect the quantitative data and WSN architecture to collect rainfall data and feeding the BRB system is illustrated in Fig. 3.

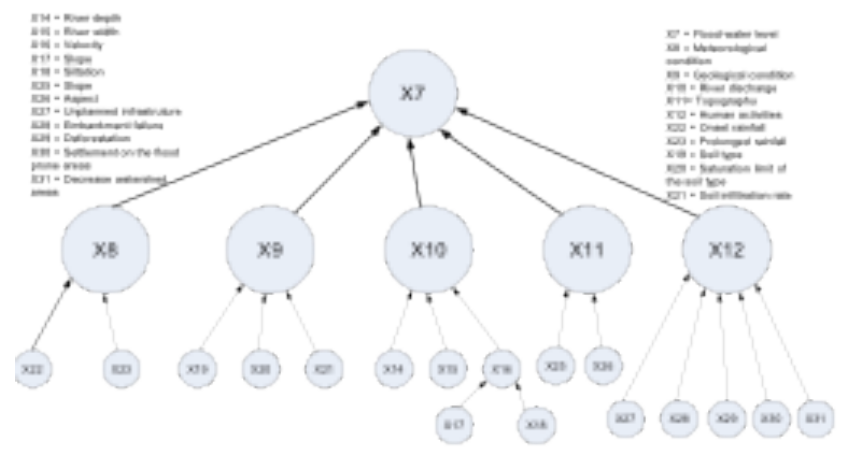

Fig. 2. BRB framework to assess flooding in an area

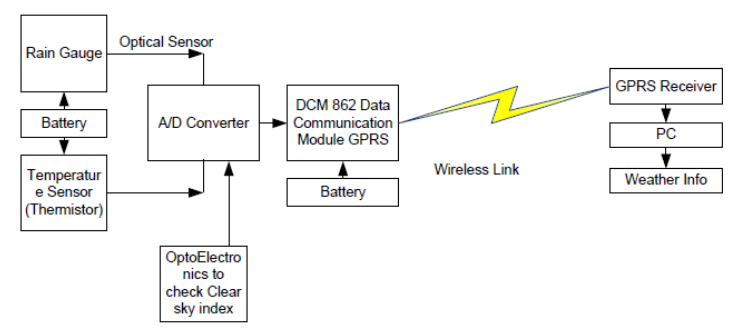

Fig. 3. WSN Architecture to collect rainfall data

\section{CASE STUdy Flood Risk Assessment System}

Fig. 4a depicts the location of the case study area, which is located in the southern part of the Chittagong district of Bangladesh and covering an area of approximately five square miles. The area consists of plain land and also with a number of canals, facilitating the drainage of rainwater. Flash flood is a regular phenomenon in this area especially during rainy season. The livelihood of the people depends upon the production of various crops in the soil of this land. Therefore, the regular prediction of flood water level or the generation flood scenarios taking different input variables into account is very essential. Table 1 shows the input data of the 17 variables for the case study area. Fig. $4 \mathrm{~b}$ illustrates the interface of the BRBES (Belief Rule Based Expert System) to predict flood water level of an area. 


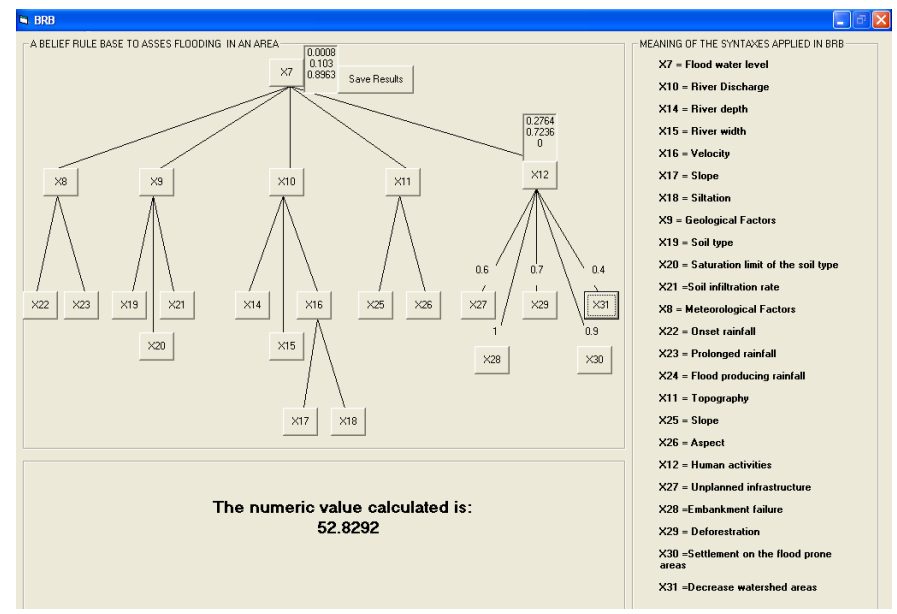

(a)

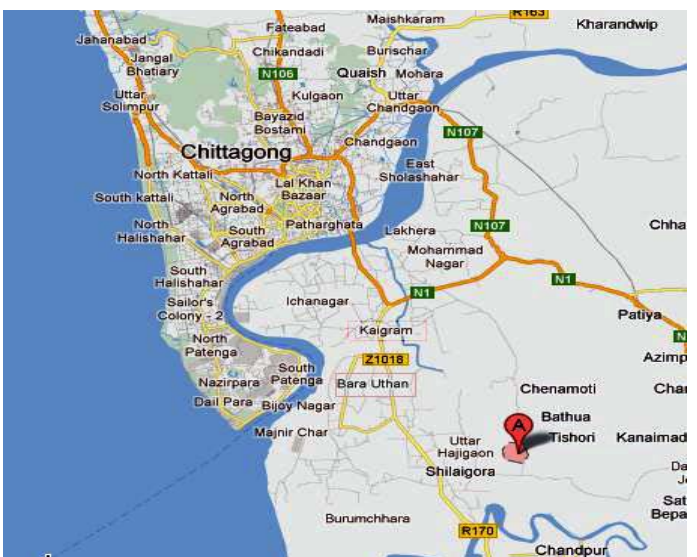

(b)

Fig. 4. a) Interface of the BRB Expert System and b) Case Study Area

The input data on the qualitative variables have been collected from the local people by taking account of their opinion, while WSN have been used to collect quantitative data, especially which are dynamic in nature such as rainfall data. However, fixed quantitative data such as slope of the area or river depth have been collected from the various private and public organizations. It is important to note that the dynamics of the flooding mainly depend on the value of the variables, which change at each point in time such as rainfall. Therefore, the employment of WSN is considered as the crucial to develop a smart risk assessment system, especially to predict flood water level in an area.

Table 1. Input data of the 17 variables for the case study area

\begin{tabular}{|c|c|c|c|c|c|c|c|c|c|c|c|c|c|c|}
\hline $\mathrm{X}$ & $\mathrm{X}$ & $\mathrm{X}$ & $\mathrm{X}$ & $\mathrm{X}$ & $\mathrm{X}$ & $\mathrm{X}$ & $\mathrm{X}$ & $\mathrm{X}$ & $\mathrm{X}$ & $\mathrm{X}$ & $\mathrm{X}$ & $\mathrm{X}$ & $\mathrm{X}$ & $\mathrm{X}$ \\
2 & 2 & 1 & 2 & 2 & 1 & 1 & 1 & 1 & 2 & 2 & 2 & 2 & 2 & 3 \\
2 & 3 & 9 & 0 & 1 & 4 & 5 & 7 & 8 & 5 & 6 & 7 & 8 & 9 & 0 \\
\hline 7 & 3 & 1 & 3 & 9 & 1 & 2 & 1 & 7 & 3 & 8 & 7 & 1 & 4 & 9 \\
5 & & & 0 & & 6 & 5 & 8 & & 6 & & & & & \\
& & & & & 0 & & & & & & & & & \\
\hline
\end{tabular}

\section{CONCLUSION AND FUTURE WORK}

We described how a smart risk assessment system using belief-rule-based expert systems and WSN technologies could be built. Building on our previous research, where models for heterogeneous wireless networks [56] were developed and also mobile e-services [57] and infrastructures for smart cities [54] were studied along with development of GIS-based flood impact assessment software, known as FIAGIS [2][6][53][55], we intend to build an experimental system implementing the solutions outlined in this article.

\section{ACKNOWLEDGMENT}

This work was partially supported by the NIMO (Nordic Interaction and Mobility Research Platform) project [57] funded by the EU Interreg IVA North program.

\section{REFERENCES}

[1] Connell et al. (2003). Climate adaptation: risk, uncertainty and decisionmaking. http://www.ukcip.org.uk/resources/publications/pub\dets.asp? ID=4, 2003, Retrieved on April 15, 2014.

[2] Hossain. (2002). An investigation into the provision of a GIS to assess risks associated with flood disasters on road transportation, MPhil Thesis, Department of Computation, UMIST, U.K.

[3] DEFRA, Flood Risk Assessment for New Development. R\\&D Technical Report FD 2320/TR2, March 2005.

[4] Huntington. (2005). Flood risk management project inception report, Flood Risks Management Research Consortium.

[5] Hall et al. (2003). Quantified scenarios analysis of drivers and impacts of changing flood risk in England and Wales: 2030-2100, Global Environmental Change Part B: Environmental Hazards 5(3-4):51-65

[6] Hossain et al. (2006). An Information System to Assess Flood Impact on Road Transportation Systems, International Journal of Computers and Their Applications 13(2):73-80

[7] Thumere et al. (2000). A GIS based coastal management system for climate change associated flood risk assessment on the east coast of England, International Journal of Geographical Information Science 14(3):265-281

[8] Hall et al. (2003). A methodology for national-scale flood risk assessment, Water and Maritime Engineering, ICE, 156(3) 235-247

[9] Nicholls. (2003). An Expert Assessment of Storm Surge "Hotspots". Final Report, Centre for Hazard and Risk research, Lamont-Doherty Observatory, Columbia University

[10] Hall. (2006). Uncertainty-based sensitivity indices for imprecise probability distribution, Reliability Engineering \& System Safety 91(1011):1443-1451

[11] Yang et al. (1994). An evidential reasoning approach for multiple attribute decision making with uncertainty, IEEE Transactions on Systems, Man, and Cybernetics 24(1):1-18

[12] Yang (2001). Rule and utility based evidential reasoning approach for multiple attributedecision analysis under uncertainty, European Journal of Operational Research, 131(1):31-61

[13] Yang et al. (2002). On the evidential reasoning algorithm for multiattribute decision analysis under uncertainty. IEEE Transactions on Systems, Man, and Cybernetics Part A: Systems and Humans, 32(3):289-304

[14] Hossain et al. (2013). An Intelligent Tender Evaluation System Using Evidential Reasoning Approach, International Journal of Computer Applications 61(15):38-43

[15] Mahmud (2012). An Evidential Reasoning-based Decision Support System to Support House Hunting, International Journal of Computer Applications 57(21):51-58

[16] Yang et al. (2006). A belief rule-base inference methodology using the evidential reasoning approach - RIMER, IEEE Transactions on Systems, Man, and Cybernetics - Part A: Systems and Humans, 36(2):266- 285 
[17] Yang et al. (2006). Optimal learning method for a new belief rule based system. IEEE Transactions on Systems, Man, and Cybernetics - Part A

[18] Angulo et al. (2012). Fuzzy expert system for the detection of episodes of poor water quality through continuous measurement. Expert Systems with Applications, 39(1), 1011-1020.

[19] Liu et al. (1996). Detection of roller bearing defects using expert system and fuzzy logic. Mechanical Systems and Signal Processing, 10(5), 595614.

[20] Russel et al. (2009). Artificial Intelligence: A Modern Approach (3rd ed.). Upper Saddle River: Prentice Hall.

[21] Hossain et al. (2013). A belief rule-based clinical decision support system to suspect heart failure from signs, symptoms and risk factors, Accepted for publication in Proc. 2nd international Conference of Informatics, electronics and Vision (IEEE Sponsored) http://iciev.org/

[22] Hossain et al. (2014) A Belief Rule Based Expert System to Assess Meditation. IEEE Technically Co-Sponsored The 2014 Internationa Conference on Computational Science and Computational Intelligencenference, March 10-13, 2014, Las vegas, USA.

[23] Rahman et al. (2013) A Belief Rule Based Expert System to Assess Asthma Suspicion. IEEE Technically Co-Sponsored $16^{\text {th }}$ International Conference on Computer and Information Technology, December, 2013, Khulna,, Bangladesh.

[24] Hossain et al. (2013) A Belief Rule Based Expert System to Assess Suspicion of Acute Coronary Syndrome (ACS) from Signs and Symptoms. IEEE Technically Co-Sponsored Science and Information Conference, October 7-9, 2013, London, UK.

[25] Bouma et al. (2005). Risk assessment and water management. Environmental Modelling \& Software 20(2):141-151

[26] Manene et al. (2005). Quantitative flood risk assessment for Polders. Reliability Engineering \& System Safety 90(2-3):229-237

[27] Pappenberger et al. (2005). Risk \& Uncertainty Tools and Implementation, FRMRC Report, Department of Environmental Science, Lancaster University.

[28] Thumere et al. (2000). A GIS based coastal management system for climate change associated flood risk assessment on the east coast of England, International Journal of Geographical Information Science 14(3):265-281

[29] Cameron et al. (2000). Flood frequency estimation under climate change (with uncertainty). Hydrology and Earth System Sciences 4(3):393-405

[30] Cinderby (1999). Geographical information systems (GIS) for participation: the future of environmental GIS? International Journal of Environmental Pollution 11(3): 304-315

[31] Pappenberger et al. (2005). Cascading model uncertainty from medium range weather forecasts (10 days) through a rainfall-runoff model to flood inundation predictions within the European Flood Forecasting System (EFFS). Hydrology and Earth System Science 9(4):381-393

[32] Akyildiz et al. (2010). Wireless Sensor Networks. Wiley.

[33] Ye et al. (2002). An energy-efficient MAC protocol for wireless sensor networks. Proc. INFOCOM 2002

[34] Jamieson et al. (2006). Sift: A MAC protocol for event-driven wireless sensor networks. Lecture Notes in Computer Science, Vol. 3868, pp. 260-275
[35] Demirkol et al. (2006). MAC protocols for wireless sensor networks: a survey. IEEE Communications Magazine 44(4):115-121

[36] Mansjshwar et al. (2001). TEEN: a routing protocol for enhanced efficiency in wireless sensor networks, Proc. IPDPS 2001

[37] Schurger et al. (2001). Energy efficient routing in wireless sensor networks, Proc. MILCOM 2001

[38] Al-Karaki (2004). Routing techniques in wireless sensor networks: a survey, IEEE Wireless Communications 11(6):6-28

[39] Akkaya et al. (2005). A survey on routing protocols for wireless sensor networks, Ad Hoc Networks 3(3), pp. 325-349

[40] Perrig et al. (2004). Security in wireless sensor networks, Communications of the ACM 47(6):53-57

[41] IEEE Computer Society IEEE 802.15.4 - Wireless Medium Access Control and Physical Layer (PHY) Specifications for Low-Rate Wireless Personal Area Networks (WPANs)

[42] Mulligan (2007). The 6LoWPAN Architecture. Proc. EmNets'07

[43] Shelby et al. (2010). 6LOWPAN: The Wireless Embedded Internet. Wiley

[44] OSGi Alliance (2011). OSGi Service Platform Core Specification Release 4, Version 4.3. http://www.osgi.org

[45] Kächele et al. (2011). nOSGi: a posix-compliant native OSGi framework, Proc. COMSWARE

[46] Schmidt et al. (2009). OSGi4C: enabling OSGi for the cloud, Proc COMSWARE

[47] Rosenberg et al. (2002). SIP: Session Initiation Protocol. IETF RFC 3261.

[48] Rosenberg (2004). A presence event package for the session initiation protocol (SIP). IETF RFC 3856

[49] Lonnfors et al. (2008). Session initiation protocol (SIP) extension for partial notification of presence information. IETF RFC 5263

[50] Schulzrinne (2006). The SIMPLE Presence and Event Architecture. Proc. COMSWARE

[51] Roychowdhury et al. (2009). Motivation for SIP as an application protocol for 6lowpan devices. Internet Draft

[52] Shelby et al. (2013). Constrained Application Protocol (CoAP), Internet Draft, draft-ietf-core-coap-18

[53] Toh et al. (2009). Health decision support for biomedical signals monitoring system over a WSN. Proc. Second International Symposium on Electronic Commerce and Security

[54] SenseSmartCity (2013). http://sensesmartcity.org, Retrieved on April 15, 2014.

[55] Hossain et al. (1998). Cybernetical Interrelationships: An Empirical Study with respect to Bangladesh Ecology. Kybernetes: The International Journal of Systems and Cybernetics 27(5):485-495

[56] Andersson (2010). On access network selection models and mobility support in heterogeneous wireless networks, $\mathrm{PhD}$ thesis, Luleå University of Technology, Sweden

[57] NIMO: Nordic Interaction and Mobility Research Platform, Available at http://www.nimoproject.org on April 15, 2014. 